\title{
An Efficient Engine for Orthogonal SE
}

\author{
Gongtao Wang, Ali Karimi \\ OSI Automation Software Systems (Beijing) Co., Ltd. \\ No.2 East 3rd Ring Road, Beijing 100027 \\ Beijing, China \\ Email: wanggt@tom.com
}

\author{
Jianming Xu \\ Jiangsu Power Transmission and Transformation \\ Corporation \\ No.58 Suyuan Avenue, Nanjing 211102 \\ Nanjing, China
}

\begin{abstract}
Orthogonal transformation has been used for State Estimation, since it is hard to solve by normal WLS method when weights of some measurements differ much from others. Though the numerical stability of orthogonal transformation has been generally accepted, it is reported that the efficiency might be another problem for Orthogonal SE. An efficient engine for orthogonal SE has been proposed in this paper, which is based upon fast Givens rotation and sparse matrix techniques. Further considerations including column \& rows ordering, block matrix, and fixed factor table are also explained in order to further improve the performance of this engine especially on large-scale systems. Performance testing has been made on a desktop computer using multiple power systems of different sizes, and the feasibility and efficiency of this engine has been verified.
\end{abstract}

Keywords - Power Systems, Orthogonal SE, Efficient Engine, Givens Transformation, Sparse Matrix, Block Matrix

\section{INTRODUCTION}

For a group of measurement data at a certain time, State Estimation (SE) is used to determine the estimated values of state variables (usually nodal voltages), and then perform bad data detection and identification. Weighted Least Square (WLS) is the fundamental method of SE, which has a good convergent capability and high quality of estimation, but needs a large quantity of computation and memory. When weights of some measurements differ from others as much as 100 multiples or more, the SE problem becomes hard to solve. The improvement forms of WLS are: (1) Fast Decoupled method, and (2) Orthogonal Transformation method. The later is chosen to be the topic of this paper trying to make it more efficient while still maintaining the stability and simplicity at the same time. The relationship between cross angle and measuring errors of two vectors is shown

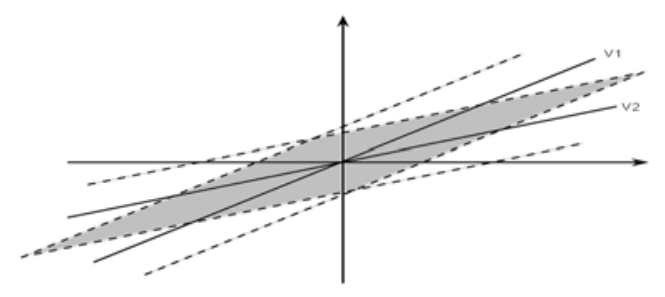

FIG 1.1 CROSS OF TWO VeCTORS WITH MEASURING

visually in Fig 1.1, where the area of shadow part denotes the error of the solution. When V1 and V2 are orthogonal, the error of the solution will become the minimum. During the past years, significant progress has been achieved on orthogonal transformation, whose numerical stability has been generally accepted compared with WLS, hybrid method, normal equations with constraints, and etc ${ }^{[1-7]}$. How to improve computational efficiency is the target of this paper.

\section{MODEL DESCRIPTION}

\section{A. WLS-Based Formulation}

Measurement equations of a measuring system can be formulated as ${ }^{[8]}$ :

$$
z=h(x)+v
$$

In which,

$$
\begin{array}{ll}
\mathrm{Z} & \text { - measurement vector } \\
\mathrm{X} & \text { - state variables } \\
\mathrm{V} & \text { - error vector } \\
\mathrm{h}(\mathrm{x}) & \text { - measurement functions }
\end{array}
$$

The objective function of state estimation, based on Weighted Least Square method, is as follows:

In which,

$$
\min J(x)=[z-h(x)]^{T} R^{-1}[z-h(x)]
$$

$$
\begin{array}{ll}
\mathrm{R}^{-1} & \text { - weights matrix } \\
\mathrm{X} & \text { - state variables }
\end{array}
$$

\section{B. Orthogonal SE}

In order to increase the numerical stability, orthogonal transformation technology is introduced into SE program. Expand the measurement equation of $\mathrm{h}(\mathrm{x})$ into Taylor series, preserving the linear term:

$$
h(x)=h\left(x_{0}\right)+H \Delta x
$$

In which, $H$ is called Jacobian matrix of measurement. And then the objective function can be rewritten as:

$$
\begin{gathered}
\min J(x)=[z-h(x)]^{T} R^{-1}[z-h(x)] \\
=R^{-1 / 2}\left[z-h\left(x_{0}\right)-H \Delta x\right]^{T} R^{-1 / 2}\left[z-h\left(x_{0}\right)-H \Delta x\right] \\
=\left[R^{-1 / 2}(r-H \Delta x)\right]^{T}\left[R^{-1 / 2}(r-H \Delta x)\right] \\
=\left|r_{w}-H_{w} \Delta x\right|^{2}
\end{gathered}
$$

Where

$r=z-h\left(x_{0}\right)$, which is called Residual Error Vector

$$
\begin{gathered}
r_{w}=R^{-1 / 2} r \\
H_{w}=R^{-1 / 2} H
\end{gathered}
$$

Supposing there is an orthogonal matrix Qmm, it brings 


$$
Q\left[\begin{array}{ll}
H_{w} & r_{w}
\end{array}\right]=\left[\begin{array}{ll}
S & b_{1} \\
0 & b_{2}
\end{array}\right]
$$

Where, $\mathrm{S}$ is an upper triangle matrix. The objective function then comes into

$$
\begin{aligned}
& J(\Delta x)=\left|r_{w}-H_{w} \Delta x\right|^{2} \\
& =\left|Q r_{w}-Q H_{w} \Delta x\right|^{2} \\
& =\left|b_{1}-S \Delta x\right|^{2}+\left|b_{2}\right|^{2}
\end{aligned}
$$

To minimize the objective function, there must be

$$
b_{1}-S \Delta x=0
$$

So $\Delta \mathrm{x}$ can be obtained by solving a group of linear equations, and the solution will be used to update the state variables.

\section{ORTHOgONAL TRANSFORMATION}

\section{A. Standard Givens Rotation}

$$
\left(\begin{array}{cc}
c & s \\
-s & c
\end{array}\right)\left(\begin{array}{l}
a \\
b
\end{array}\right)=\left(\begin{array}{l}
\hat{a} \\
0
\end{array}\right)
$$

where

$$
\begin{aligned}
& c=\frac{a}{\sqrt{a^{2}+b^{2}}} \\
& s=\frac{b}{\sqrt{a^{2}+b^{2}}}
\end{aligned}
$$

For a 2-row matrix

$$
\begin{aligned}
& A=\left(\begin{array}{lllll}
a_{1} & a_{2} & a_{3} & \ldots & a_{n} \\
b_{1} & b_{2} & b_{3} & \ldots & b_{n}
\end{array}\right) \\
& \hat{A}=\left(\begin{array}{ccccc}
\hat{a}_{1} & \hat{a}_{2} & \hat{a}_{3} & \ldots & \hat{a}_{n} \\
0 & \hat{b}_{2} & \hat{b}_{3} & \ldots & \hat{b}_{n}
\end{array}\right)
\end{aligned}
$$

Where,

$$
\begin{gathered}
\hat{a}_{j}=c a_{j}+s b_{j} \quad(j=1,2, \ldots n) \\
\hat{b}_{j}=-s a_{j}+c b_{j}
\end{gathered}
$$

One element is eliminated a time, until there are no nonzero elements on the left side of the diagonal line. Since there needs four times of multiplication for an iteration, the standard Givens rotation is also called 4-multiplication scheme.

\section{B. Fast Givens Rotation}

Let us assume for a moment that $\mathrm{c} \neq 0$. Then the Givens rotator $\mathrm{Q}$ can be factorized as

$$
\left(\begin{array}{cc}
c & s \\
-s & c
\end{array}\right)=\left(\begin{array}{cc}
c & 0 \\
0 & c
\end{array}\right)\left(\begin{array}{cc}
1 & t \\
-t & 1
\end{array}\right)
$$

where $\mathrm{t}=\mathrm{s} / \mathrm{c}$ (the $\mathrm{c}$-transformation). If $\mathrm{c}=0$ (or very small), the s-transformation can be used instead:

where $\mathrm{k}=\mathrm{c} / \mathrm{s}$

$$
\left(\begin{array}{cc}
c & s \\
-s & c
\end{array}\right)=\left(\begin{array}{cc}
s & 0 \\
0 & s
\end{array}\right)\left(\begin{array}{cc}
1 & k \\
-k & 1
\end{array}\right)
$$

Consider an $\mathrm{m} \times \mathrm{n}$ matrix $\mathrm{B}$ with full rank and $\mathrm{m}>\mathrm{n}$. A sequence of rotations can be used to triangularize $\mathrm{B}$ as indicated below:

$$
\left(\begin{array}{ccccc}
b_{11} & b_{12} & b_{13} & \ldots & b_{1 n} \\
b_{21} & b_{22} & b_{23} & \ldots & b_{2 n} \\
b_{31} & b_{32} & b_{33} & \ldots & b_{3 n} \\
\ldots & \ldots & \ldots & \ldots & \ldots \\
b_{n 1} & b_{n 2} & b_{n 3} & \ldots & b_{n n} \\
\ldots & \ldots & \ldots & \ldots & \ldots \\
b_{m 1} & b_{m 2} & b_{m 3} & \ldots & b_{m n}
\end{array}\right) \Rightarrow\left(\begin{array}{ccccc}
u_{11} & u_{12} & u_{13} & \ldots & u_{1 n} \\
0 & u_{22} & u_{23} & \ldots & u_{2 n} \\
0 & 0 & u_{33} & \ldots & u_{3 n} \\
\ldots & \ldots & \ldots & \ldots & \ldots \\
0 & 0 & 0 & \ldots & u_{n n} \\
\ldots & \ldots & \ldots & \ldots & \ldots \\
0 & 0 & 0 & \ldots & 0
\end{array}\right) .
$$

For a certation row to be rotated, fast Givens can be performed by either $c$ - or $s$-transformation, provided the orginal matrix to be factorized has a full rank.

The $c$-transformation can be formulated as

$$
\begin{aligned}
& b_{i l}=b_{i l}+p b_{j l} \\
& b_{j l}=b_{j l}-q b_{i l}
\end{aligned}
$$

And the s-transformation is formulated as follows

$$
\begin{aligned}
& b_{i l}=p b_{i l}+b_{j l} \\
& b_{j l}=q b_{j l}-b_{i l}
\end{aligned}
$$

Where, $p$ and $q$ are intermediate variables, and detailed description can be found in the reference $e^{[9,10]}$.

\section{FURTHER CONSIDERATIONS}

\section{A. Column Ordering}

Since the columns of Jacobian matrix are corresponding to state variables, i.e. nodal voltages, the Minimum Degree criterion should be followed for column ordering, so that there will be less nonzero fill-in's during orthogonal transformation. Additionally, columns for voltage magnitude and angle should be arranged alternately, in order to keep the sparsity on the upper-left part of the matrix.

\section{B. Rows Ordering}

The scheme of dynamic pivot selection is suggested to deal with dynamic nonzero fill-in in the process of factorization. For i-step shown in Fig 4.1, the row with minimum number of nonzeros is selected as the pivot row, and then all the rows below with nonzero bji will be rotated by ascending order of number of nonzero elements.
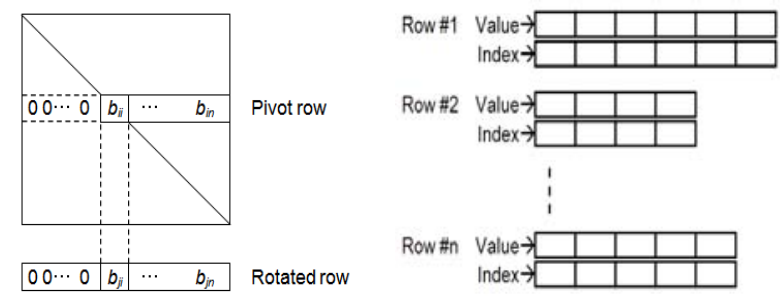

Fig 4.1 Rotation OF A Row Fig 4.2 StruCture OF SPARSE MATriX

\section{Sparse Matrix}

A structure of sparse matrix ${ }^{[1]]}$ has been designed for large-scale sparse matrix storage and operation, as shown in Fig 4.2. Basically, there are two compact vectors for each row of the matrix, which are Value and Index for nonzero elements only (either original or new fill-in), whose memory has been allocated dynamically. All the addresses of rows 
are saved in another vector with the name of the matrix to make it easy and efficient to use.

\section{Block Matrix}

With the development of orthogonal rotations, the lowerright part of matrix is becoming denser more and more. Taking the 14 nodes system as an example, the changing process on the sparsity of Jacobi matrix is shown in Fig 4.3. Numerical experiments on large systems showed that orthogonal operation will be slowing down distinctly when it comes to 6000 and more steps. Inspired by the thought of block decomposition ${ }^{[12,13]}$, the lower-right part is regarded as a dense submatrix and rotated seperately until the upperright triangular matrix is formed.

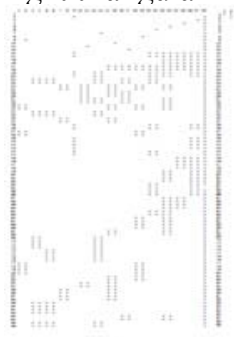

(a)

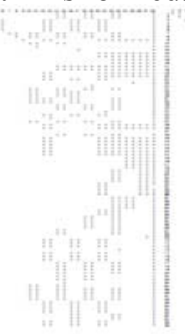

(b)

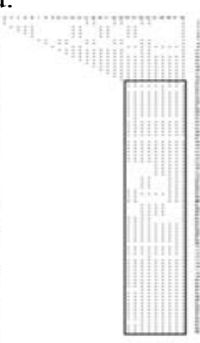

(c) (d)

Fig 4.3 EVOLUTION OF THE SPARSE MATrix

(a) The Original Sparse Matrix

(b) The Early Stage of Orthogonal Transformation

(c) The Later Stage, Dense/Block Matrix formed

(d) The Final Triangular Matrix

\section{E. Fixed Factor Table}

Usually there will be multiple iterations of orthogonal transformation to reach a convergent SE solution. To improve the performance further, the weighted Jacobi matrix $\mathrm{Hw}$ in equation (2-5) can be factorized into a Fixed Factor Table saving the information of the whole rotation process, which is just similar to that in Fast Decoupled Power Flow. For the varying residual error vector in certain iteration, only a single fast forward \& back substitution is necessary to obtain the solution of voltage correction. Additionally, orthogonal SE converges on a solution after fewer iterations ${ }^{[14]}$.

\section{Testing Cases}

A. System Information

\begin{tabular}{|l|c|c|c|}
\hline & Case \#4 & Case \#5 & Remarks \\
\hline Node_num & 3,898 & 15,166 & \\
\hline Branche_num & 5,441 & 18,591 & \\
\hline Nonxf_branch & 4,263 & 12,421 & \\
\hline Xformer_num & 1,142 & 5,764 & \\
\hline Gener_num & 789 & 2,970 & \\
\hline Load_num & 3,069 & 7,606 & \\
\hline Shunt_num & 23 & 805 & \\
\hline DCline_num & 0 & 1 & \\
\hline Multi_dc & 0 & 2 & \\
\hline
\end{tabular}

\begin{tabular}{|c|c|c|c|}
\hline PIJ_measNum & 5,441 & 18,241 & \multirow{2}{*}{ for all branches } \\
\hline QIJ_measNum & 5,441 & 18,241 & \\
\hline PI_measNum & 1,943 & 7,870 & \multirow{4}{*}{$\begin{array}{l}\text { for all nonzero } \\
\text { injection nodes }\end{array}$} \\
\hline QI_measNum & 1,943 & 7,870 & \\
\hline VV_measNum & 1,943 & 7,870 & \\
\hline VA measNum & 0 & 0 & \\
\hline
\end{tabular}

\section{B. Performance Testing}

Performance testing has been made on a desktop computer, whose machine information is Dell Optiplex 990 (2011) with Intel CPU i5-2400@3.10GHz and 3.23GB memory. Running time is counted to finish a complete orthogonal transformation on Jacobian matrix based on several power systems of different sizes. Considering the high redundancy of measurements, three schemes of measurement configuration, which are Branches-only, Branches \& Nodal Voltages, and Full Measurements, have been applied to Cases 4 and 5 respectively. The performance of this engine is summarized as follows (Fig 5.1), which shows an approximate linear trend.

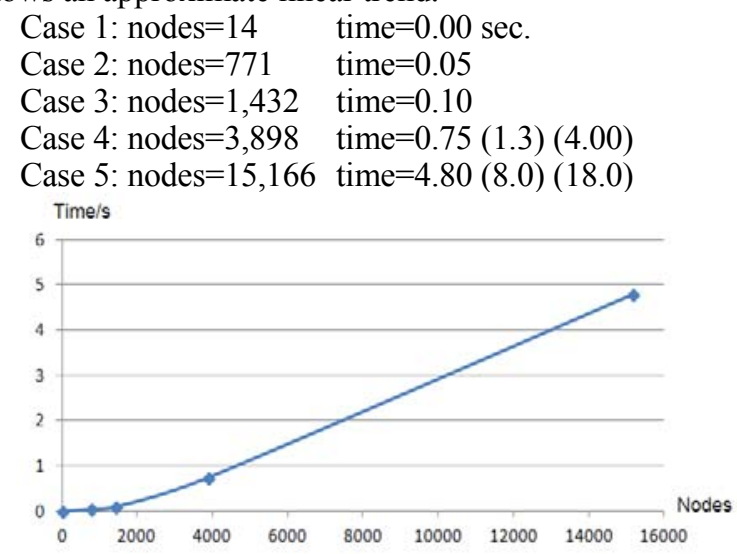

FIg 5.1 PERFormance ON DIFFERENT SySTEMS

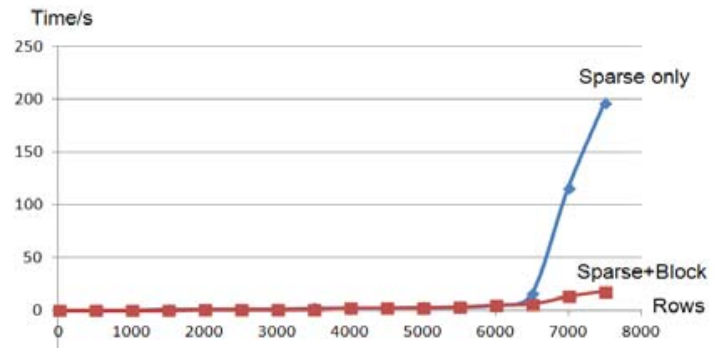

Fig 5.2 EFFECt OF Block Matrix TeChNiQue

A specific testing case has also been made on 4000 nodes model, to show the effect of block matrix technique, especially when orthogonal rotation comes to 6000 rows and more, as shown in Fig 5.2. In order to show the advantage of the engine proposed in this paper, a comparison has been made on performance of various testing cases from both the references and our own resource, as shown in Tab 5.1. 
TAB 5.1 COMPARISON ON TESTING CASES

\begin{tabular}{|r|r|r|r|r|r|l|}
\hline Year & Nodes & \multicolumn{1}{|c|}{ Meas. } & Redun. & Nonzero & Time [s] & Remark \\
\hline 1985 & 118 & 540 & 2.30 & N/A & $1.0-2.2$ & Hybrid $^{[2]}$ \\
\hline 1985 & 99 & 542 & 2.75 & $1500+$ & $1.0-3.1$ & Hachtel $^{[3]}$ \\
\hline 1999 & 1,044 & 7,194 & 3.45 & 35,720 & 10.0 & FastGivens $^{[7]}$ \\
\hline \multirow{4}{*}{2008} & 300 & 1,000 & 1.67 & 4,000 & 8.8 & \multirow{2}{*}{ arallel } \\
\cline { 1 - 6 } & 600 & 2,000 & 1.67 & 8,000 & 76.9 & \multirow{2}{*}{ QR $^{[15]}$} \\
\cline { 2 - 6 } & 1,200 & 4,000 & 1.67 & 16,000 & 738.0 & \\
\hline \multirow{4}{*}{2013} & 14 & 96 & 3.56 & 452 & 0.00 & \\
\cline { 2 - 6 } & 771 & 3,758 & 2.44 & 18,420 & 0.05 & \multirow{2}{*}{ This paper } \\
\cline { 2 - 6 } & 1,432 & 5,063 & 1.77 & 27,121 & 0.10 & \\
\cline { 2 - 6 } & 3,898 & 10,882 & 1.40 & 50,786 & 0.75 & \\
\cline { 2 - 6 } & 15,166 & 36,482 & 1.20 & 162,159 & 4.80 & \\
\hline
\end{tabular}

\section{CONCLUSION}

An efficient engine for orthogonal SE has been proposed, which is based upon fast Givens rotation and sparse matrix techniques. Further considerations including column \& rows ordering, block matrix, and fixed factor table, are also explained in order to improve the performance of the engine especially on large-scale systems. Performance testing has been made on a desktop computer using power systems of different sizes, and the feasibility and efficiency of this engine has been verified. This orthogonal SE/engine can works as an alternative function where equations are solved in normal WLS product.

\section{REFERENCE}

[1] Simoes-Costa A., Quintana V., An Orthogonal Row Processing Algorithm for Power System Sequential State Estimation, IEEE Trans. on Power Apparatus and Systems, Vol.100, No.8, August 1981

[2] Monticelli A., Murari C., etc., A Hybrid State Estimator: Solving Normal Equations By Orthogonal Transformations, IEEE Trans. on Power Apparatus and Systems, Vol.104, No.12, Dec 1985

[3] A. Gjelsvik, S. Aam, etc., Hachtel's Augmented Matrix Method - A Rapid Method Improving Numerical Stability in Power System Static State Estimation, Paper 85 WM026-0, IEEE PES Winter Meeting 1985

[4] Holten L., Gjelsvik A., etc., Comparison of Different Methods for State Estimation, IEEE Trans. on Power Systems, Vol.3, No.4, Nov 1988

[5] N. Vempati, Ilya W. Slutsker, Enhancements To Givens Rotations For Power System State Estimation, IEEE Trans. on Power Systems, VoI.6, No.2, May 1991

[6] N. Vempati, Ilya W. Slutsker, etc., Orthogonal Sparse Vector Methods, Trans. on Power SysIems, Vol.7, No.2, May 1992

[7] Pandian, K. Parthasarathy, etc., Towards Faster Givens Rotations Based Power System State Estimator, IEEE Trans. on Power Systems, Vol.14, No.3, August 1999

[8] Yu Erkeng, "Power System State Estimation", Water and Electric Power Press, 1985

[9] Monticelli, "State Estimation in Electric Power Systems - A Generalized Approach”, Kluwer Academic Publishers, 1999

[10] Ali Abur, Antonio Gomez Exposito, "Power System State Estimation Theory and Implementation", CRC Press Taylor \& Francis Group, 2004

[11] Wang Xifan, Fang Wanliang, etc., "Modern Power System Analysis", Science Press, 2003
[12]Du Zhengchun, Niu Zhenyong, etc., A Block QR Based Power System State Estimation Algorithm, Proceedings of the CSEE, 2003.08

[13] Guo Ruipeng, Shao Xuejian, etc., A Block Givens Rotations Based Algorithm for Power System State Estimation, Proceedings of the CSEE, 2006.06

[14]Dinesh Salem-Natarajan, Mani Subramanian, etc., State Estimator for CA ISO Market and Security Applications, IEEE PES General Meeting 2008

[15]Fredrik Nysjo, Per Uddholm, Parallel QR-Factorisation for Power System State Estimation,Report in Scientific Computing Advanced Course, May 2008

\section{DISCUSSION WITH ALI}

1. References ${ }^{[5,6]}$ have extensively investigated the effect of sparse vector method and the enhanced version of Givens rotation in addition to row ordering schemes that speed up solutions of power network equations.

Tom: Only when the solution is a sparse vector, in other words bus voltages on a part of the power system (island) needs to be modified, the sparse vector method applies. As for column \& row ordering schemes, Minimum Degree (MD) and Increasing order of row count (R1) are used in this paper.

2. Hybrid state estimation has been proposed ${ }^{[2]}$. In their approach they combined numerical robust orthogonal method with desirable sparsity feature close to WLS. Description and the implementation of a fast version of the Givens rotations are presented for orthogonal state estimation $^{[7]}$.

Tom: As an enhancement to WLS, the hybrid method handles those ill-conditioning equations by orthogonal transformation. But a simple and separate orthogonal SE/engine based on fast Givens is involved in this paper.

3. What is the novel approach and contribution of this paper?

Tom: An orthogonal SE/engine based upon fast Givens transformation has been described in this paper, where fundamental techniques on sparse matrix, block matrix, and etc. are used to realize this engine. The better efficiency has been proven by testing cases compared with the results of previous studies.

4. Case study is not clearly explained on block matrix. More detail specification is required for the large complex system, if there is any novel approach is presented.

Tom: As you know, the lower-right part of the matrix will become much denser with the progress of orthogonal transformation, so it is an efficient and simple way to handle this submatrix as a traditional dense/full matrix. Additional information has been added to Section 5.2.

5. Figure 4.3, sparse matrix does not have any citation or reference system.

Tom: It is drawn to show the changing process of the sparse Jacobi matrix, which comes from an internal test-ing system of 14 nodes given by OSI US.

6. Although the graph shows improvement, but there is no comparison provided for the systems with same number of nodes.

Tom: Additional information on comparison has been added to Section 5.2

7. The research should show not only the concerns with the speed of orthogonal state estimation versus normal 
form of WLS, but also the connectivity of state estimation core engine with observability and bad data analysis.

Tom: In system integration, orthogonal SE/engine works as an alternative function where equations are solved in normal WLS product. All the peripheral functions are shared including observability analysis and bad data detection. Related information has been added to Section 4.6. 